\title{
Analysis of an impulsive pest management SEI model with nonlinear incidence rate
}

\author{
XIA WANG and XINYU SONG \\ College of Mathematics and Information Science, Xinyang Normal University \\ Xinyang 464000 Henan, P.R. China \\ E-mail: xywangxia@163.com
}

\begin{abstract}
According to biological strategy for pest control, we investigate the dynamic behavior of a pest management SEI model with nonlinear incidence concerning impulsive strategyperiodic releasing infected pests at fixed times. We prove that all solutions of the system are uniformly ultimately bounded and there exists a globally asymptotically attractive pest-eradication periodic solution when the impulsive period satisfies $A_{1}$. When the impulsive period satisfies $A_{2}$, the stability of pest-eradication periodic solution is lost, the system is uniformly permanent. Thus, we can use the stability of the positive periodic solution and its period to control insect pests at acceptably low levels.
\end{abstract}

Mathematical subject classification: $34 \mathrm{C} 05,92 \mathrm{D} 25$.

Key words: pest-management, boundedness, global attractivity, permanence, extinction.

\section{Introduction}

The health and socioeconomic risks posed by severe and sudden epidemics of infectious disease like SARS, or the assessed impact of a potential influenza pandemic, or a measles pre and post-eradication outbreaks, are compelling scientists to design and implement more effective control and preparedness programs. Pulse vaccination is an effective method to use in attempts to control infectious diseases. The pulse vaccination strategy (PVS) consists of periodical repetitions

\#CAM-51/09. Received: 07/II/09. Accepted: 18/II/09.

Correspondence to: Xia Wang 
of impulsive vaccinations in a population in contrast to the traditional constant vaccination. At each vaccination time, a constant fraction of susceptible population is vaccinated. This vaccination is called impulsive when all the vaccine doses are applied in a very short span of time. PVS allows to reach the eradication of a disease with some practical advantages, as discussed in $[1,18,19]$.

Over the last fifty years, epidemic models have been received a great attention in mathematical ecology. Various types of SIR epidemic model were studied by Anderson and May [11-13] in the context of microbic infections on the dynamics of animal populations. SIR epidemic models assume that the disease incubation period is negligible so that each susceptible individual becomes infectious and later recovers with a permanently or temporarily acquired immunity. Unlike SIR models, SEI models suppose that a susceptible individual first goes through a latent (exposed) period before becoming infectious.

For IPM strategy, we combine the biological control and chemical control. The infectious pests are released periodically every time period $\tau$, meanwhile periodic spraying the microbial pesticide for susceptible pests. The infected pests have the function similar to the microbial pesticide and can infect the healthy pests, further weaken or disable their prey function till death. Based on biological control strategy in pest management, we construct a pest-epidemic model with impulsive control. An alternative to chemical control is biological control, which is generally, man's use of specially chosen living organism, referred as the biocontrol agent, to control another. Biological control agents can be predator, pathogens or parasites of the organism to be controlled that either kills the harmful organism or interferes with its biological processes $[2-3,5,7-8,10]$. For example, Asian Tiger Mosquito can transmit viruses which cause dengue fever, Ross River fever or Japanese encephalitis. To avoid this and to control the spread of mosquito swarms, we could spray with Bti, which is a variety of the bacterium Bacillus thuringiensis (Bt). Also, Insects, like humans and other animals, can be infected by disease-causing organisms such as bacteria, viruses and fungi. Under appropriate conditions, such as high humidity or high pest abundance, these naturally occurring organisms may multiply to cause disease outbreaks or epizootics that can decimate an insect population. This kind of method obtains its target in two ways. In the first way, a small pathogen is introduced in a pest 
population with expectation that it will generate an epidemic which will persist at an endemic level. For the second one, an insect pathogen is used like biopesticides. In this case, it is applied whenever a pest population is at an economically significant level and there is no expectation that the pathogen persists in the environment for a long time. An advantage of using insect pathogens is that they are safe to men and are usually safe to beneficial insects. Conversely, biological control features effective control on pests with an improved ecosystem, lower cost and without any pollution.

Impulsive differential equations found in almost of the dynamics of applied science and have been studied in many investigations $[6,9,12,14,20]$. But to our knowledge there are only a few paper and books on mathematical model of the dynamics of microbial diseases in pest control. Anderson et al. pointed out that standard incidence is more suitable than bilinear incidence [13]. Levin et al. have adopted a incidence form like $\beta S^{q} I^{p}$ or $\frac{\beta S^{q} I^{p}}{N}$ which depends on different infective diseases and environment $[16,17]$. In the following, we shall examine the use of pathogens in a more flexible manner. The main feature of the present paper is to study the dynamic behavior of the model we construct and obtain some conditions under which the pest becomes eradicable or not. The pest-epidemic model with impulses, i.e., periodic releasing infected pests at fixed moments is described as follows:

$$
\left\{\begin{array}{l}
\dot{S}(t)=r S(t)\left(1-\frac{S(t)}{K}\right)-\beta S(t) I^{q}(t), \\
\dot{E}(t)=\beta S(t) I^{q}(t)-(\alpha+\mu) E(t), \\
\dot{I}(t)=\alpha E(t)-\mu I(t), \\
\Delta S(t)=0, \\
\Delta E(t)=0, \\
\Delta I(t)=p .
\end{array}\right\} t=n \tau, n \in \mathrm{Z}_{+},
$$

where $S(t), E(t)$ and $I(t)$ are densities of susceptible, exposed, infectious pests at time $t$, respectively. $S(t)$ is in the absence of $I(t)$ grows logistically with carrying capacity $K$, and with an intrinsic birth rate constant $r, \beta S(t) I^{q}(t)$ is the contact rate, $\alpha$ is the inverse of latent period. $\mu$ is the death rate of the exposed 
and infectious pests, and $r, K, \beta, \mu, p, q$ are positive constants. $p$ is the release amount of the infected pests at $t=n \tau, n \in \mathrm{Z}_{+}, Z_{+}=\{0,1,2, \cdots\},, \tau$ is the period of the impulsive effect. $\Delta S(t)=S\left(t^{+}\right)-S(t), \Delta E(t)=E\left(t^{+}\right)-$ $E(t), \Delta I(t)=I\left(t^{+}\right)-I(t), f\left(t^{+}\right)=\lim _{x \rightarrow t^{+}} f(x)$. That is, we can use releasing infected pests to eradicate pests or keep the pest population below the damage level.

\section{Notations and definitions}

In this section, we agree on some notations which will prove useful and give some definitions.

Let $R_{+}=[0, \infty), R_{+}^{3}=\left\{x \in R^{3}: x>0\right\}, \Omega=$ int $R_{+}^{3}, Z_{+}$be the set of all nonnegative integers. Denote $f=\left(f_{1}, f_{2}, f_{3}\right)$, the map defined by the right hand side of the first two equations of system (1.1). Let $V_{0}=\left\{V: R_{+} \times R_{+}^{3} \mapsto R_{+}\right\}$, continuous on $(n \tau,(n+1) \tau] \times R_{+}^{3}$, and $\lim _{(t, y) \rightarrow\left(n \tau^{+}, x\right)} V(t, y)=V\left(n \tau^{+}, x\right)$ exists.

Definition 2.1. $V \in V_{0}$, then for $V(t, x) \in(n \tau,(n+1) \tau] \times R_{+}^{3}$, the upper right derivative of $V(t, x)$ with respect to the impulsive differential system (1.1) is defined as

$$
D^{+} V(t, x)=\lim _{h \rightarrow 0} \sup \frac{1}{h}[V(t+h, x+h f(t, x))-V(t, x)] .
$$

The solution of system (1.1) is a piecewise continuous function $x: R_{+} \mapsto R_{+}^{3}$, $x(t)$ is continuous on $(n \tau,(n+1) \tau], n \in \mathrm{Z}_{+}$and $x\left(n \tau^{+}\right)=\lim _{t \rightarrow n \tau^{+}} x(t)$ exists. Obviously the smoothness properties of $f$ guarantee the global existence and uniqueness of solution of system (1.1), for details see [4, 15].

We will use a basic comparison result from impulsive differential equations. For convenience, we state it in our notations.

Suppose $g: R_{+} \times R_{+} \mapsto R$ satisfies:

(H) $g$ is continuous in $(n \tau,(n+1) \tau] \times R_{+}$and for $x \in R^{+}, n \in Z_{+}$, $\lim _{(t, y) \rightarrow\left(n \tau^{+}, x\right)} g(t, y)=g\left(n \tau^{+}, x\right)$ exists. 
Lemma 2.1. Let $V \in V_{0}$, assume that

$$
\begin{cases}D^{+} V(t, x) \leq g(t, V(t, x)), & t \neq n \tau, \\ V\left(t, x\left(t^{+}\right)\right) \leq \psi_{n}(V(t, x(t))), & t=n \tau,\end{cases}
$$

where $g: R_{+} \times R_{+} \mapsto R$ satisfies $(\mathrm{H})$ and $\psi_{n}: R_{+} \mapsto R_{+}$is nondecreasing. Let $r(t)$ be the maximal solution of the scalar impulsive differential equation

$$
\left\{\begin{array}{l}
\dot{u}(t)=g(t, u(t)), \quad t \neq n \tau, \\
u\left(t^{+}\right)=\psi_{n}(u(t)), \quad t=n \tau, \\
u\left(0^{+}\right)=u_{0},
\end{array}\right.
$$

existing on $[0, \infty)$. Then $V\left(0^{+}, x_{0}\right) \leq u_{0}$ implies that $V(t, x(t)) \leq r(t), t \geq 0$, where $x(t)$ is any solution of (1.1), similar result can be obtained when all the directions of the inequalities in the lemma are reversed and $\psi_{n}$ is nonincreasing. Note that if we have some smoothness conditions of $g$ to guarantee the existence and uniqueness of solutions for (2.2), then $r(t)$ is exactly the unique solution of (2.2).

Lemma 2.2. Suppose that $x(t)$ is a solution of (1.1) with $x\left(0^{+}\right) \geq 0$, then $x(t) \geq 0$ for $t \geq 0$, and further if $x\left(0^{+}\right)>0$, then $x(t)>0$ for all $t>0$.

For convenience, we give some basic properties of the following system:

$$
\left\{\begin{array}{l}
\dot{u}(t)=a-b u(t), \quad t \neq n \tau, \\
\Delta u(t)=p, \quad t=n \tau, \\
u\left(0^{+}\right)=u_{0} \geq 0 .
\end{array}\right.
$$

We have the following lemma:

Lemma 2.3. System (2.3) has a unique positive periodic solution $\widetilde{u}(t)$ with period $\tau$ and for every solution $u(t)$ of (2.3) such that $|u(t)-\widetilde{u}(t)| \rightarrow 0$ as $t \rightarrow \infty$, where

$$
\begin{aligned}
\tilde{u}(t) & =\frac{a}{b}+\frac{p \exp (-b(t-n \tau))}{1-\exp (-b \tau)}, t \in(n \tau,(n+1) \tau], n \in Z_{+}, \\
\widetilde{u}\left(0^{+}\right) & =\frac{a}{b}+\frac{p}{1-\exp (-b \tau)}
\end{aligned}
$$

and $\tilde{u}(t)$ is globally asymptotically stable. 
Proof. Clearly $\widetilde{u}(t)$ is a positive periodic solution of (2.3) with period $\tau$ when $a \neq 0$. Integrating and solving the first equation of (2.3) between pulses, we get

$$
u(t)=\frac{a}{b}+\left(u\left(n \tau^{+}\right)-\frac{a}{b}\right) \exp (-b(t-n \tau)), t \in(n \tau,(n+1) \tau] .
$$

After each successive pulse, we can deduce the following stroboscopic map of system (2.4)

$$
u\left((n+1) \tau^{+}\right)=\frac{a}{b}+\left(u\left(n \tau^{+}\right)-\frac{a}{b}\right) \exp (-b \tau)+p .
$$

Equation (2.5) has a unique fixed point $u^{*}=\frac{a}{b}+\frac{p}{1-e^{-b \tau}}$, it corresponds to the unique positive periodic solution $\widetilde{u}(t)$ of system $(2.3)$, the initial value $\widetilde{u}\left(0^{+}\right)=$ $u^{*}=\frac{a}{b}+\frac{p}{1-e^{-b \tau}}$. The fixed point $u^{*}$ of the stroboscopic map implies that there is a corresponding cycle of period $\tau$ in $u(t)$, that is $\widetilde{u}(t)=\frac{a}{b}+\frac{p \exp (-b(t-n \tau))}{1-\exp (-b \tau)}$, $t \in(n \tau,(n+1) \tau]$ using iterative technique on $(2.5)$, we obtain

$$
u\left(n \tau^{+}\right)=\left(p+\frac{a}{b}(1-\exp (-b \tau))\right) \frac{1-\exp (-n b \tau)}{1-\exp (-b \tau)}+u\left(0^{+}\right) \exp (-n b \tau),
$$

thus, $u\left(n \tau^{+}\right) \rightarrow u^{*}$ as $n \rightarrow \infty$, so $\widetilde{u}(t)$ is globally asymptotically stable. And thus, we have

$$
u(t)=\left(u\left(0^{+}\right)-\widetilde{u}\left(0^{+}\right)\right) \exp (-b t)+\widetilde{u}(t) .
$$

Therefore, $u(t) \rightarrow \widetilde{u}(t)$ as $t \rightarrow \infty$, that is $|u(t)-\widetilde{u}(t)| \rightarrow 0$ as $t \rightarrow \infty$.

If $a=0$, the system (2.3) has a unique positive periodic solution $\widetilde{u}(t)=$ $\frac{p \exp (-b(t-n \tau))}{1-\exp (-b \tau)}$ and with initial value $\tilde{u}\left(0^{+}\right)=\frac{p}{1-\exp (-b \tau)}$ and $\tilde{u}(t)$ is globally asymptotically stable. The proof is complete.

Therefore, system (1.1) has a pest-eradication periodic solution $(0,0, \widetilde{I}(t))$, where $\widetilde{I}(t)=\frac{p \exp (1-\mu(t-n \tau))}{1-\exp (-\mu \tau)}$.

\section{Stability of the pest-eradication periodic solution}

In this section, we study the stability of the pest-eradication periodic solution as a solution of the full system (1.1). Firstly, we present the Floquet theory for the linear $\tau$-periodic impulsive equation

$$
\begin{cases}\frac{d x}{d t}=A(t) x, & t \neq \tau_{k}, t \in R, \\ \Delta x=B_{k} x, & t=n \tau_{k}, k \in Z_{+} .\end{cases}
$$


Then we introduce the following conditions:

$\left(H_{1}\right) A(\cdot) \in P C\left(R, C^{n \times n}\right)$ and $A(t+\tau)=A(t)(t \in R)$,

$\left(H_{2}\right) \quad B_{k} \in C^{n \times n}, \operatorname{det}\left(E+B_{k}\right) \neq 0, \tau_{k}<\tau_{k+1}\left(k \in Z_{+}\right)$,

$\left(H_{3}\right)$ There exists a $h \in Z_{+}$, such that $B_{k+h}=B_{k}, \tau_{k+h}=\tau_{k}+\tau\left(k \in Z_{+}\right)$.

Let $\Phi(t)$ be a fundamental matrix of (3.1), then there exists a unique nonsingular matrix $M \in C^{n \times n}$ such that:

$$
\Phi(t+\tau)=\Phi(t) M(t \in R) .
$$

By equality (3.2) there corresponds to the fundamental matrix $\Phi(t)$ the constant matrix $M$ which we call the monodromy matrix of (3.1) (corresponding to the fundamental matrix of $\Phi(t)$ ). All monodromy matrices of (3.1) are similar and have the same eigenvalues. The eigenvalues $\lambda_{1}, \lambda_{2}, \cdots, \lambda_{n}$ of the monodromy matrices are called the Floquet multipliers of (3.1).

Lemma 3.1. (Floquet theory). Let conditions $\left(\mathrm{H}_{1}-\mathrm{H}_{3}\right)$ hold. Then the linear $\tau$ periodic impulsive equation (3.1) is

(1) stable if and only is all multipliers $\lambda_{j}(j=1,2, \cdots, n)$ of equation (3.1) satisfy the inequality $\left|\lambda_{j}\right| \leq 1$ and, moreover, to those $\lambda_{j}$ for which $\left|\lambda_{j}\right|=1$ there correspond simple elementary divisors.

(2) asymptotically stable if and only if all multipliers $\lambda_{j}(j=1,2, \cdots, n)$ of equation (3.1) satisfy the inequality $\left|\lambda_{j}\right|<1$.

(3) unstable if $\left|\lambda_{j}\right|>1$ for some $j=1,2, \cdots, n$.

Lemma 3.2. There exists a positive constant $M$ such that $S(t) \leq M ; E(t) \leq$ $M ; I(t) \leq M$, for each solution $(S(t), E(t), I(t))$ of system (1.1) with positive initial values, where $t$ is large enough.

Proof. Let

$$
V(t)=S(t)+E(t)+I(t) .
$$


Then $V(t) \in V_{0}$ and the upper right derivative of $V(t)$ along a solution of (1.1) is described as

$$
\left.D^{+} V(t)\right|_{(1.1)}+\mu V(t)=(r+\mu) S(t)-\frac{r S^{2}(t)}{K} \leq L_{0},
$$

where $L_{0}=\frac{K(r+\mu)^{2}}{4 r}$, when $t=n \tau$, we obtain

$$
V\left(n \tau^{+}\right)=V(n \tau)+p
$$

According to Lemma 2.2, we have

$$
\begin{aligned}
V(t, x(t)) \leq & V\left(0^{+}\right) \exp (-\mu t)+\int_{0}^{t} L_{0} \exp (-\mu(t-s)) \mathrm{d} s \\
& +\sum_{0<n \tau<t} p \exp \left(\int_{n \tau}^{t}(-\mu) \mathrm{d} s\right) \\
\leq & V\left(0^{+}\right) \exp (-\mu t)+\frac{L_{0}}{\mu}(1-\exp (-\mu t)) \\
& +\frac{p \exp (-\mu(t-\tau))}{1-\exp (\mu \tau)}+\frac{p \exp (\mu \tau)}{\exp (\mu \tau)-1} \\
\rightarrow & \frac{L_{0}}{\mu}+\frac{p \exp (\mu \tau)}{\exp (\mu \tau)-1}, t \rightarrow \infty .
\end{aligned}
$$

Therefore $V(t)$ is ultimately bounded by a constant and there exists a constant $M>0$, such that $S(t) \leq M, E(t) \leq M, I(t) \leq M$ for each solution $x(t)=$ $(S(t), E(t), I(t))$ of (1.1) with all $t$ large enough.

Theorem 3.1. The pest-eradication periodic solution $(0,0, \widetilde{I}(t))$ is globally asymptotically stable provided

$$
\mathrm{A}_{1}: r \tau<\frac{p^{q} \beta(1-\exp (-q \mu \tau))}{q \mu[1-\exp (-\mu \tau)]^{q}} .
$$

Proof. Firstly, we prove the local stability of a $\tau$-period solution $(0,0, \widetilde{I}(t))$ may be determined by considering the behavior of small-amplitude perturbations $(x(t), y(t), z(t))$ of the solution.

Define

$$
S(t)=x(t), \quad E(t)=y(t), \quad I(t)=z(t)+\widetilde{I}(t),
$$


where $x(t), y(t), z(t)$ are small perturbations, there may be written as

$$
\left(\begin{array}{l}
x(t) \\
y(t) \\
z(t)
\end{array}\right)=\Phi(t)\left(\begin{array}{c}
x(0) \\
y(0) \\
z(0)
\end{array}\right),
$$

where $\Phi(t)$ satisfy

$$
\frac{d \Phi(t)}{d t}=\left(\begin{array}{ccc}
r-\beta \widetilde{I}^{q}(t) & 0 & 0 \\
\beta \widetilde{I}^{q}(t) & -(\mu+\alpha) & 0 \\
0 & \alpha & -\mu
\end{array}\right) \Phi(t)
$$

with $\Phi(0)=I$, where $I$ is the identity matrix. The resetting impulsive conditions of (1.1) becomes

$$
\left(\begin{array}{l}
x\left(n \tau^{+}\right) \\
y\left(n \tau^{+}\right) \\
z\left(n \tau^{+}\right)
\end{array}\right)=\left(\begin{array}{lll}
1 & 0 & 0 \\
0 & 1 & 0 \\
0 & 0 & 1
\end{array}\right)\left(\begin{array}{l}
x(n \tau) \\
y(n \tau) \\
z(n \tau)
\end{array}\right) .
$$

Hence, if absolute values of all eigenvalues of

$$
M=\left(\begin{array}{lll}
1 & 0 & 0 \\
0 & 1 & 0 \\
0 & 0 & 1
\end{array}\right) \Phi(\tau)=\Phi(\tau),
$$

are less than one, the $\tau$-periodic solution is locally stable. By calculating, we have

$$
\Phi(\tau)=\left(\begin{array}{ccc}
\exp \left(\int_{0}^{\tau}\left(r-\beta \widetilde{I}^{q}(t)\right) \mathrm{d} t\right) & 0 & 0 \\
* & \exp (-(\alpha+\mu) \tau) & 0 \\
* & * & \exp (-\mu \tau)
\end{array}\right),
$$

there is no need to calculate the exact form of $(*)$ as it is not required in the analysis that follows. Then the eigenvalues of $M$ denoted by $\lambda_{1}, \lambda_{2}$ and $\lambda_{3}$ are the following:

$$
\begin{aligned}
& \lambda_{1}=\exp \left(\int_{0}^{\tau}\left(r-\beta \widetilde{I}^{q}(t)\right) \mathrm{d} t\right), \\
& \lambda_{2}=\exp (-(\alpha+\mu) \tau)<1, \\
& \lambda_{3}=\exp (-\mu \tau)<1,
\end{aligned}
$$


it follows that $\left|\lambda_{1}\right|<1$ if and only if condition $A_{1}$ holds true. According to Lemma 3.1 , the pest-eradication solution $(0,0, \widetilde{I}(t))$ is locally asymptotically stable.

In the following, we prove the global attractivity. Choose sufficiently small $\varepsilon>0$ such that

$$
\delta=\exp \left(\int_{0}^{\tau}\left(r-\beta(\widetilde{I}(t)-\varepsilon)^{q}\right) \mathrm{d} t\right)<1 .
$$

From the first equation of system (1.1), we obtain

$$
\dot{S}(t) \leq r S(t)\left(1-\frac{S(t)}{K}\right) .
$$

Consider the following impulsive differential equation:

$$
\begin{cases}\dot{u}(t)=r u(t)\left(1-\frac{u(t)}{K}\right), & t \neq n \tau, \\ \Delta u(t)=S(0), & t=n \tau,\end{cases}
$$

we have $S(t) \leq u(t)$ and $u(t) \rightarrow K$ as $t \rightarrow \infty$. Thus, there exists a $\xi>0$, such that $S(t) \leq K+\xi$ for $t$ large enough. Without loss of generality, we assume $S(t) \leq K+\xi$ for all $t>0$. Noting that $\dot{I}(t) \geq-\mu I(t)$, consider the following impulsive differential equation:

$$
\begin{cases}\dot{v}(t)=-\mu v(t), & t \neq n \tau, \\ \Delta v(t)=p, & t=n \tau .\end{cases}
$$

By Lemma 2.3, system (3.7) has a globally asymptotically stable positive periodic solution

$$
\widetilde{v}(t)=\frac{p \exp (1-\mu(t-n \tau))}{1-\exp (-\mu \tau)}=\widetilde{I}(t), \quad n \tau<t \leq(n+1) \tau .
$$

So by Lemma 2.2, we get

$$
I(t) \geq v(t)>\widetilde{I}(t)-\varepsilon .
$$

From system (1.1), we obtain that

$$
\begin{cases}\dot{S}(t) \leq S(t)\left(r-\beta(\widetilde{I}(t)-\varepsilon)^{q}\right), & t \neq n \tau, \\ \Delta S(t)=0, & t=n \tau .\end{cases}
$$


Integrating (3.9) on $(n \tau,(n+1) \tau]$, which yields

$$
S\left((n+1) \tau^{+}\right) \leq S(n \tau) \exp \left(\int_{n \tau}^{(n+1) \tau}\left(r-\beta(\widetilde{I}(t)-\varepsilon)^{q}\right) \mathrm{d} t\right)=S(n \tau) \delta .
$$

Thus, $S(n \tau) \leq S\left(0^{+}\right) \delta^{n}$ and $S(n \tau) \rightarrow 0$ as $n \rightarrow \infty$. Therefore, $S(t) \rightarrow 0$ as $t \rightarrow \infty$, since $0<S(t)<S(n \tau) \delta$ for $n \tau<t \leq(n+1) \tau$.

If $\lim _{t \rightarrow \infty} S(t)=0$, for $\varepsilon_{0}>0$ small enough, there exists a $t_{1}>0$ such that $0<S(t)<\varepsilon_{0}$ for $t>t_{1}$. It is obviously by Lemma 3.2, for a sufficiently small $\varepsilon_{1}>0$, there exists a $t_{2}>t_{1}$, such that $\beta S(t) I^{q}(t)<\varepsilon_{1}$ for $t>t_{2}$. From the second equation of system (1.1), we have

$$
\dot{E}(t) \leq \varepsilon_{1}-(\mu+\alpha) E(t),
$$

we obtain that

$$
E(t) \leq E\left(0^{+}\right) \exp (-(\mu+\alpha) t)+\frac{\varepsilon_{1}}{\mu+\alpha}-\frac{\varepsilon_{1}}{\mu+\alpha} \exp (-(\mu+\alpha) t) \rightarrow 0
$$

as $t \rightarrow \infty$ and $\varepsilon_{1} \rightarrow 0$. Therefore, $E(t) \rightarrow 0$ as $t \rightarrow \infty$.

Next, we prove that $I(t) \rightarrow \widetilde{I}(t)$ as $t \rightarrow \infty$, for sufficiently small $\varepsilon_{2}>0$, there exists a $t_{3}>t_{2}>0$ such that $0<E(t)<\varepsilon_{2}$ for all $t>t_{3}$. From system (1.1), we have

$$
\begin{cases}\dot{I}(t) \leq \alpha \varepsilon_{2}-\mu I(t), & t \neq n \tau, \\ \Delta I(t) \leq p, & t=n \tau,\end{cases}
$$

considering the following comparison system

$$
\begin{cases}\dot{z}(t)=\alpha \varepsilon_{2}-\mu z(t), & t \neq n \tau, \\ \Delta z(t)=p, & t=n \tau .\end{cases}
$$

By lemma 2.3, system (3.13) has a positive periodic solution

$$
\widetilde{z}(t)=\frac{p \exp (-\mu(t-n \tau))}{1-\exp (-\mu \tau)}+\frac{\alpha \varepsilon_{2}}{\mu}, n \tau<t \leq(n+1) \tau,
$$

which is globally asymptotically stable. Thus, for sufficiently small $\varepsilon_{3}$, such that

$$
I(t) \leq z(t)<\widetilde{z}(t)+\varepsilon_{3} .
$$

Combining (3.8) and (3.14), we obtain $\widetilde{I}(t)-\varepsilon<I(t)<\widetilde{z}(t)+\varepsilon_{3}$ for $t$ large enough, let $\varepsilon, \varepsilon_{3} \rightarrow 0$, we get $\widetilde{z}(t) \rightarrow \widetilde{I}(t)$, then $I(t) \rightarrow \widetilde{I}(t)$ as $t \rightarrow \infty$. This completes the proof. 


\section{Permanence}

Definition 4.1. System (1.1) is said to be uniformly persistent if there is an $l>0$ (independent of the initial conditions) such that every solution $(S(t)$, $E(t), I(t))$ of system (1.1) satisfies

$$
\lim _{t \rightarrow \infty} \inf S(t) \geq l, \lim _{t \rightarrow \infty} \inf E(t) \geq l, \lim _{t \rightarrow \infty} \inf I(t) \geq l .
$$

Definition 4.2. System (1.1) is said to be permanent if there exists a compact region $\Gamma \subset$ int $R_{+}^{3}$ such that every solution $(S(t), E(t), I(t))$ of system (1.1) will eventually enter and remain the region $\Gamma$.

Theorem 4.1. If

$$
\mathrm{A}_{2}: r \tau>\frac{p^{q} \beta(1-\exp (-q \mu \tau))}{q \mu[1-\exp (-\mu \tau)]^{q}}
$$

holds, then there exists a positive constant $\tilde{m}_{1}$ such that every positive solution $(S(t), E(t), I(t))$ of system (1.1) satisfies $S(t) \geq \tilde{m}_{1}$ for $t$ large enough.

Proof. In the following, we will prove that there exists a constant $m_{1}>0$, such that $S(t)>m_{1}$ for $t$ large enough. From system (1.1), we can see $\dot{I}(t)>-\mu I(t)$, then consider the comparison system

$$
\begin{cases}\dot{I}(t)=-\mu I(t), & t \neq n \tau, \\ \Delta I(t)=p, & t=n \tau .\end{cases}
$$

According to Lemma 2.2 and Lemma 2.3, we obtain for any $\epsilon>0, I(t)>$ $\widetilde{I}(t)-\epsilon$ for $t$ large enough. So, if we can find positive number $\widetilde{m}_{1}>0$ such that $S(t) \geq \tilde{m}_{1}$ for $t$ large enough, then our aim is reached. We will do it in the following two steps for convenience.

Step I: If $r \tau>\frac{p^{q} \beta(1-\exp (-q \mu \tau))}{q \mu[1-\exp (-d \tau)]^{q}}$ holds true, we can choose $0<m_{1}<K r$ and $\epsilon^{\prime}$ small enough such that

$$
\delta_{1}=\exp \left(\int_{n \tau}^{(n+1) \tau}\left(r-\frac{m_{1}}{K}-\beta\left(\widetilde{I}(t)+\epsilon^{\prime}\right)^{q}\right) \mathrm{d} t\right)>1,
$$


we will prove there exist a $t_{1} \in(0, \infty)$, such that $S\left(t_{1}\right) \geq m_{1}$. Otherwise $S(t)<m_{1}$ for all $t>0$. From system (1.1), we obtain that

$$
\begin{cases}\dot{I}(t) \leq \alpha M-\mu I(t), & t \neq n \tau, \\ \Delta I(t)=p, & t=n \tau,\end{cases}
$$

consider the following comparison system

$$
\begin{cases}\dot{w}(t) \leq \alpha M-\mu w(t), & t \neq n \tau, \\ \Delta w(t)=p, & t=n \tau,\end{cases}
$$

by Lemmas 2.2 and 2.3 on (4.3), there exists a $T_{1}>0$ such that $I(t) \leq w(t)<$ $\widetilde{w}(t)+\epsilon^{\prime}$ for $t>T_{1}$, where

$$
\widetilde{w}(t)=\frac{\alpha M}{\mu}+\frac{p \exp (-\mu(t-n \tau))}{1-\exp (-\mu \tau)}, t \in(n \tau,(n+1) \tau] .
$$

Thus, from system (1.1) we obtain that

$$
\dot{S}(t) \geq\left(r-\frac{m_{1}}{K}-\beta\left(\widetilde{w}(t)+\epsilon^{\prime}\right)^{q}\right) S(t) .
$$

Let $n_{1} \in Z_{+}$and $n_{1} \tau>T_{1}$, integrating (4.4) on $(n \tau,(n+1) \tau], n \geq n_{1}$, then we obtain

$$
\begin{aligned}
S((n+1) \tau) & \geq S(n \tau) \exp \left(\int_{n \tau}^{(n+1) \tau}\left(r-\frac{m_{1}}{K}-\beta\left(\widetilde{w}(t)+\epsilon^{\prime}\right)^{q}\right) \mathrm{d} t\right) \\
& =S(n \tau) \delta_{1} .
\end{aligned}
$$

Then $S\left(\left(n_{1}+k\right) \tau\right) \geq S\left(n_{1} \tau\right) \delta_{1}{ }^{k}, k \rightarrow \infty$, which is a contradiction to the boundedness of $S(t)$. Hence there exists a $t_{1}>0$ such that $S\left(t_{1}\right) \geq m_{1}$.

Step II: If $S(t) \geq m_{1}$ for all $t \geq t_{1}$, then our aim is obtained. Otherwise $S(t)<m_{1}$ for some $t \geq t_{1}$, setting $t^{*}=\inf _{t>t_{1}}\left\{S(t)<m_{1}\right\}$, we have $S(t) \geq m_{1}$ for $t \in\left[t_{1}, t^{*}\right)$ and $t^{*} \in\left(n_{1} \tau,\left(n_{1}+1\right) \tau\right], n_{1} \in Z_{+}$. It is easy to see $S\left(t^{*}\right)=m_{1}$ since $S(t)$ is continuous. Select $n_{2}, n_{3} \in Z_{+}$such that

$$
n_{2} \tau>-\frac{1}{\mu} \ln \frac{\epsilon^{\prime}}{M+p}, \quad \exp \left(\eta\left(n_{2}+1\right) \tau\right) \delta_{1}{ }^{n_{3}}>1,
$$


where $\eta=r-\frac{m_{1}}{K}-\beta M^{q}<0$. We claim that there must exists a $t^{\prime} \in\left(\left(n_{1}+\right.\right.$ 1) $\left.\tau,\left(n_{1}+1\right) \tau+T\right]$ such that $S(t) \geq m_{1}$, otherwise $S(t)<m_{1}, t \in\left(\left(n_{1}+\right.\right.$ 1) $\left.\tau,\left(n_{1}+1\right) \tau+T\right]$, consider (4.3) with $w\left(\left(n_{1}+1\right) \tau^{+}\right)=S\left(\left(n_{1}+1\right) \tau^{+}\right)$, we have

$$
w(t)=\left(w\left(\left(n_{1}+1\right) \tau^{+}\right)-\frac{\alpha M}{\mu}-\frac{p}{1-\exp (-\mu \tau)}\right) \exp (-\mu t)+\widetilde{w}(t),
$$

for $t \in(n \tau,(n+1) \tau], n_{1}+1 \leq n \leq n_{1}+1+n_{2}+n_{3}$. Then $|w(t)-\widetilde{w}(t)|<$ $(M+p) \exp \left(-\mu\left(t-\left(n_{1}+1\right) \tau\right)\right)<\epsilon^{\prime}$, and $w(t) \leq I(t)<\widetilde{w}(t)+\epsilon^{\prime}$ for $\left(n_{1}+1+n_{2}\right) \tau \leq t \leq\left(n_{1}+1\right) \tau+T$, which implies (4.4) holds for $\left(n_{1}+n_{2}+1\right) \tau \leq t \leq\left(n_{1}+1\right) \tau+T$, system (1.1) gives

$$
S\left(\left(n_{1}+1+n_{2}+n_{3}\right) \tau\right) \geq S\left(\left(n_{1}+1+n_{2}\right) \tau\right) \delta_{1}{ }^{n_{3}} .
$$

There are the following two cases for $t \in\left(t^{*},\left(n_{1}+1\right) \tau\right]$ :

Case 1: If $S(t)<m_{1}$ for $t \in\left(t^{*},\left(n_{1}+1\right) \tau\right]$, then $S(t)<m_{1}$ for all $t \in$ $\left(t^{*},\left(n_{1}+1+n_{2}\right) \tau\right]$. System (1.1) gives

$$
\dot{S}(t) \geq\left(r-\frac{m_{1}}{K}-\beta M^{q}\right) S(t)=\eta S(t) .
$$

Integrating system (4.5) on this interval $t \in\left(t^{*},\left(n_{1}+1+n_{2}\right) \tau\right]$, which yields $S\left(\left(n_{1}+1+n_{2}\right) \tau\right) \geq m_{1} \exp \left(\eta\left(n_{2}+1\right) \tau\right)$. Then we have $S\left(\left(n_{1}+1+n_{2}+n_{3}\right) \tau\right) \geq$ $m_{1} \exp \left(\eta\left(n_{2}+1\right) \tau\right) \delta_{1}{ }^{n_{3}}>m_{1}$, which is a contradiction.

Let $\bar{t}=\inf _{t>t^{*}}\left\{S(t) \geq m_{1}\right\}$, then $S(\bar{t})=m_{1}$ and (4.5) holds for $t \in\left[t^{*}, \bar{t}\right)$. Integrating (4.5) on $t \in\left[t^{*}, \bar{t}\right)$

$$
S(t) \geq S\left(t^{*}\right) \exp \left(\eta\left(t-t^{*}\right)\right) \geq m_{1} \exp \left(\eta\left(1+n_{2}+n_{3}\right) \tau\right):=\tilde{m}_{1} .
$$

For $t>\bar{t}$, the same arguments can be continued since $S(\bar{t}) \geq m_{1}$. Hence $S(t) \geq \widetilde{m}_{1}$ for all $t>t_{1}$.

Case 2: If there exists a $t^{\prime \prime} \in\left(t^{*},\left(n_{1}+1\right) \tau\right]$ such that $S\left(t^{\prime \prime}\right) \geq m_{1}$. Let $\hat{t}=\inf _{t>t^{*}}\left\{S(t) \geq m_{1}\right\}$, then $S(t)<m_{1}$ for $t \in\left[t^{*}, \hat{t}\right)$ and $S(\hat{t})=m_{1}$. For $t \in\left[t^{*}, \hat{t}\right)$, (4.5) holds and integrating (4.5) on $\left[t^{*}, \hat{t}\right)$, we have

$$
S(t) \geq S\left(t^{*}\right) \exp \left(\eta\left(t-t^{*}\right)\right) \geq m_{1} \exp (\eta \tau)>\tilde{m}_{1} .
$$


This process can be continued since $S(\hat{t}) \geq m_{1}$, and we have $S(t) \geq \tilde{m}_{1}$ for $t>t_{1}$. Thus in both cases, we conclude $S(t) \geq \tilde{m}_{1}$ for all $t>t_{1}$. The proof is completed.

Theorem 4.2. Suppose $r \tau>\frac{p^{q} \beta(1-\exp (-q \mu \tau))}{q \mu[1-\exp (-d \tau)]^{q}}$, then the system (1.1) is permanent.

Proof. Denote $(S(t), E(t), I(t))$ as any solution of the system (1.1), we get

$$
\dot{I}(t) \geq-\mu I(t) .
$$

By the same argument as those in the proof of Theorem 4.1, we have

$$
\lim _{t \rightarrow \infty} I(t) \geq \widetilde{m}_{3}
$$

where

$$
\tilde{m}_{3}=\frac{p \exp (-\mu \tau)}{1-\exp (-\mu \tau)}-\varepsilon .
$$

In view of Theorem 4.1, the second equation of system (1.1) becomes

$$
\dot{E}(t) \geq \beta \widetilde{m}_{1} \tilde{m}_{3}^{q}-(\alpha+\mu) E(t) .
$$

It is easy to obtain $\lim _{t \rightarrow \infty} E(t) \geq \tilde{m}_{2}$, where $\tilde{m}_{2}=\frac{\beta \widetilde{m}_{1} \tilde{m}_{3}^{q}}{(\alpha+\mu)}-\varepsilon$. Let

$$
\Gamma=\left\{(S, E, I): S \geq \tilde{m}_{1}, E \geq \tilde{m}_{2}, I \geq \tilde{m}_{3}, S+E+I \leq M\right\} .
$$

By Theorem 4.1 and above discussion, we know that the set $\Gamma \subset$ int $R_{+}^{3}$ is global attractor, i.e., every solution of system (1.1) will eventually enter and remain in region $\Gamma$. Therefore, system (1.1) is permanent. This completes the proof.

\section{Conclusion}

In this paper, we have investigated the dynamic behavior of a pest management SEI model with nonlinear incidence and periodic releasing infected pests at fixed times. The infected pests have the function similar to the microbial pesticide and can infect the healthy pests, further weaken or disable their prey function till death. We have shown that there exists an asymptotically stable the pest-eradication periodic solution if $r \tau<\frac{p^{q} \beta(1-\exp (-q \mu \tau))}{q \mu[1-\exp (-\mu \tau)]^{q}}$. However, from 
a pest control point of view, our aim is to keep pests at acceptably low levels, not to eradicate them, only to control their population size. Therefore, when $r \tau>\frac{p^{q} \beta(1-\exp (-q \mu \tau))}{q \mu[1-\exp (-\mu \tau)]^{q}}$, the stability of pest-eradication periodic solution is lost, system (1.1) is permanent.

Acknowledgments. This work is supported by the National Natural Science Foundation of China (No. 10771179), and program for Innovative Research Team (in Science and Technology) in University of Henan Province and Innovation Scientists and Technicians Troop Construction Projects of Henan Province, the Young Backbone Teacher Foundation of Xinyang Normal University.

\section{REFERENCES}

[1] A. D'Onofrio, Stability properties of pulse vaccination strategy in SEIR epidemic model. Math. Biosci., 179 (2002), 57-72.

[2] A.J. Cherry, C.J. Lomer and F. Schulthess, Pathogen incidence and their potential as microbial control agents in IPM of maize stemborers in West Africa. Biocontrol, 44(a) (1999), 301-327.

[3] B. Liu, Z. Teng and L. Chen, Analysis of a predator-prey model with Holling II functional response concerning impulsive control strategy. J. Comput. Appl. Math., 193 (2006), 347-362.

[4] D.D. Bainov and P.S. Simeonov, System with Impulse Effect, Theory and Applications. Ellis Harwood series in Mathematics and its Applications, Chichester: Ellis Harwood (1993).

[5] H.J. Freedman, Graphical stability, enrichment, and pest control by a natural enemy. Math. Biosci., 31 (1976), 207-225.

[6] H. Zhang and L.S. Chen, Pest management through continuous and impulsive control strategies. BioSystems, 90 (2007), 350-361.

[7] J.C. Van Lenteren, Measures of success in biological control of arthropods by augmentation of natural enemies, in: S., Wratten, and G. Gurr, (Eds.), Measures of Success in Biological Control (2000).

[8] J. Grasman, O.A. Van Herwarrden, L. Hemerik et al., A two-component model of hostparasitoid interactions: Determination of the size of inundative releases of parasitoids in biological pest control. Math. Biosci., 196 (2001), 207-216.

[9] J.J. Jiao and L.S. Chen, A pest management SI model with periodic biological and chemical control concern. Appl. Math. Comput., 183 (2006), 1018-1026.

[10] M.L. Luff, The potential of predators for pest control. Agri. Ecos. Environ., 10 (1983), 159-181. 
[11] R.M. Anderson and R.M. May, Population biology of infectious diseases: Part I. Nature, 280 (1979), 361-367.

[12] R. Anderson and R. May, Population Biological of Infections Diseases. Springer, Berlin, Heidelberg, New York (1982).

[13] R. Anderson and R. May, Infectious Diseases of Human: Dynamics and Control. Oxford University Press (1991).

[14] S.Y. Tang and L.S. Chen, Density-dependent birth rate, birth pulses and their population dynamic consequences. J. Math. Biol., 44 (2002), 185-199.

[15] V. Lakshmikantham, D.D Bainov and P.S. Simeonov, Impulsive Differential Equations: Periodic Solutions and Applications. Pitman Monographs and Surreys in Pure and Applied Mathematics, 66 (1993).

[16] W.M. Liu, S.A. Levin and Y. Lwasa, Influence of nonlinear incidence rates upon the behavior of SIRS Epidemiological models. J. Math. Biol., 25 (1987), 359-380.

[17] W.M. Liu, H.W. Hethcote and S.A. Levin, Dynamical behavior of epidemiological models with nonlinear incidence rates. J. Math. Biol., 23 (1986), 187-240.

[18] X. Wang and X.Y. Song, Mathematical models for the control of a pest population by infected pest. Computers and Mathematics with Applications, 56 (2008), 266-278.

[19] Z.H. Lu, X.B. Chi and L.S. Chen, The effect of constant and pulse vaccination on SIR epidemic model with horizontal and vertical transmission. Math. Comput. Model., 36 (2002), $1039-1057$.

[20] Z. Agur, L. Cojocaru, R. Anderson and Y. Danon, Pulse mass measles vaccination across age cohorts. Proc. Natl Acad. Sci. USA, 90 (1993), 11698-11702. 